\title{
New equation of state involving Bose-Einstein condensate of antikaon for supernova and neutron star merger simulations
}

\author{
Tuhin Malik ${ }^{1}$, Sarmistha Banik ${ }^{1}$, and Debades Bandyopadhyay ${ }^{2}$ \\ 1 Birla Institute of Technology and Science, Pilani, Hyderabad Campus, Hyderabad - 500078, India \\ 2 Saha Institute of Nuclear Physics, HBNI, 1/AF Bidhannagar, Kolkata-700064, India
}

Received: date / Revised version: date

\begin{abstract}
We compute a new equation of state table including Bose-Einstein condensate of $K^{-}$mesons for core collapse supernova and neutron star merger simulations. Nuclei and interacting nucleons in nonuniform matter is described in an extended version of the nuclear statistical equilibrium model including excluded volume effects whereas the uniform matter at higher densities is treated in the relativistic hadron field theory with density dependent couplings. The equation of state table is generated for a wide range of density $\left(10^{-12}\right.$ to $\left.\sim 1 \mathrm{fm}^{-3}\right)$, positive charge fraction (0.01 to 0.60$)$ and temperature (0.1 to $\left.158.48 \mathrm{MeV}\right)$. The impact of antikaon condensate is investigated on different thermodynamic quantities for example free energy per baryon, entropy per baryon, pressure as well as compositions of matter. Furthermore, critical temperatures of antikaon condensation and the phase diagram of matter are also studied in this article.
\end{abstract}

PACS. 9 7.60.Jd neutron stars - 26.60.Kp equations of state

\section{Introduction}

Equation of state (EoS) of matter is an important microphysical input for core collapse supernova (CCSN) and binary neutron star (BNS) merger simulations. Many EoSs were developed based on updated knowledge derived from nuclear physics experimental data and neutron star observations $[1,2,3,4,5,6,7,8,9,10,11,12,13$. One such widely used nuclear EoS known as Hempel and Schaffner (HS) EoS describes the inhomogeneous matter within the framework of the nuclear statistical equilibrium (NSE) model whereas the uniform dense nuclear matter is computed in the finite temperature relativistic mean field (RMF) model with and without density dependent (DD) couplings 1, 14. The nuclear EoS table adopting the virial expansion for a non-ideal gas of nucleons and nuclei was computed by Shen et al. 22. The nuclear EoS was also constructed in a variational calculation using Argonne v18 (AV18) and Urbana IX (UIX) bare nuclear forces 4,5. The nuclear EoSs mentioned above are compatible with $2 \mathrm{M}_{\odot}$ neutron stars. The first supernova EoS table that exploited measured masses and radii of neutron stars, was developed by Steiner and collaborators 3 .

Neutron star observations could provide important inputs in the construction of EoS tables for CCSN and BNS merger simulations. Neutron star masses in relativistic binaries have been estimated to very high degree of precision owing to measurements of post-Keplerian parameters of

Correspondence to: sarmistha.banik@hyderabad.bitspilani.ac.in pulsars such as orbital decay, periastron advance, Shapiro delay, time dilation. Several heavy neutron stars of masses $\sim 2 M_{\odot}$ were discovered in the past decade. The first among those was PSR 0348+0432 having mass $2.01 \pm 0.04$ $\mathrm{M}_{\odot}$ [15. Now the millisecond pulsar PSR $0740+6620$ is credited as the most massive neutron star of $2.14_{-0.09}^{+0.10} \mathrm{M}_{\odot}$ [16. Recently Neutron Star Interior Composition Explorer (NICER) has reported the first simultaneous determination of mass and radius of PSR J0030+0451 17. This puts a stringent constraint on the $\beta$-equilibrated EoS of neutron star matter.

It has been long debated that additional degrees of freedom in the form of strange baryons [18, quarks [13] or Bose-Einstein condensate of antikaons [19,20] might appear in dense matter. Many model calculations involving hyperon matter, quark matter, Bose-Einstein condensate of antikaons, showed that the EoS of $\beta$-equilibrated matter might result in $2 \mathrm{M}_{\odot}$ or more massive neutron stars [18,21, 22 . Several temperature dependent EoS tables including exotic matter such as quark and hyperon matter were constructed and employed in CCSN and BNS merger simulations $[23,24,25,26,27,28,29,30,31,32,33$. These EoS tables are functions of wide range of values of three parameters - baryon density, positive charge fraction and temperature. A few of those temperature dependent EoS tables with exotic matter such as Banik, Hempel and Bandyopadhyay $\Lambda$-hyperons $\operatorname{EoS}(\mathrm{BHB} \Lambda \phi)$ where hyperon-hyperon interaction is mediated by $\phi$ mesons, was directly compatible with the $2 \mathrm{M}_{\odot}$ neutron star [31]. 
So far, there is no EoS table involving Bose-Einstein condensate of antikaons for CCSN and BNS merger simulations. This motivates us to construct an EoS table with antikaon condensate. The paper is organised as follows. We describe the EoS with an antikaon condensate within the NSE and field theoretical models in Section 2. Results are discussed in Section 3. We conclude in Section 4.

\section{Equation of State}

The inhomogeneous matter is described by HS EoS [1]. In this case, the ensemble of nuclei and nucleons are treated in an extended version of the NSE model using the RMF model for interacting nucleons. It also considers excluded volume effects in the thermodynamically consistent manner and excited states of light nuclei. The medium modification of nuclei due to screening of Coulomb energies of background electrons are taken into account [1]. Finally the sub-saturation density matter was matched with the dense uniform matter.

We adopt a density dependent relativistic hadron field theory for the description of strongly interacting dense baryonic matter. Here nucleon-nucleon interaction is mediated by exchanges of scalar $\sigma$, vector $\omega$ and $\rho$ mesons. This is given by the Lagrangian density [31,34,

$$
\begin{aligned}
\mathcal{L}_{N}= & \sum_{N} \bar{\psi}_{N}\left(i \gamma_{\mu} \partial^{\mu}-m_{N}+g_{\sigma N} \sigma-g_{\omega N} \gamma_{\mu} \omega^{\mu}\right. \\
& \left.-g_{\rho N} \gamma_{\mu} \boldsymbol{\tau}_{N} \cdot \boldsymbol{\rho}^{\mu}\right) \psi_{N} \\
& +\frac{1}{2}\left(\partial_{\mu} \sigma \partial^{\mu} \sigma-m_{\sigma}^{2} \sigma^{2}\right)-\frac{1}{4} \omega_{\mu \nu} \omega^{\mu \nu} \\
& +\frac{1}{2} m_{\omega}^{2} \omega_{\mu} \omega^{\mu}-\frac{1}{4} \boldsymbol{\rho}_{\mu \nu} \cdot \boldsymbol{\rho}^{\mu \nu}+\frac{1}{2} m_{\rho}^{2} \boldsymbol{\rho}_{\mu} \cdot \boldsymbol{\rho}^{\mu} .
\end{aligned}
$$

Here $\psi_{N}$ stands for nucleon doublet, $\tau_{N}$ is the isospin operator and density dependent meson-nucleon couplings are denoted by $g_{\alpha N}$ with $\alpha$ representing meson fields.

The grand-canonical thermodynamic potential per unit volume of the nuclear phase is given by

$$
\begin{aligned}
\frac{\Omega}{V}= & \frac{1}{2} m_{\sigma}^{2} \sigma^{2}-\frac{1}{2} m_{\omega}^{2} \omega_{0}^{2}-\frac{1}{2} m_{\rho}^{2} \rho_{03}^{2}-\Sigma^{r} \sum_{i=n, p} n_{i} \\
& -2 T \sum_{i=n, p} \int \frac{d^{3} k}{(2 \pi)^{3}}\left[\ln \left(1+e^{-\beta\left(E^{*}-\nu_{i}\right)}\right)\right. \\
& \left.+\ln \left(1+e^{-\beta\left(E^{*}+\nu_{i}\right)}\right)\right],
\end{aligned}
$$

where the temperature is defined as $\beta=1 / T$, effective nucleon mass $m_{N}^{*}=m_{N}-g_{\sigma N} \sigma$ and $E^{*}=\sqrt{\left(k^{2}+m_{N}^{* 2}\right)}$. We calculate all thermodynamic quantities such as pressure $P=-\Omega / V$, energy density and entropy density. The chemical potential is given by The nucleon chemical potential $\left(\mu_{N}\right)$ is defined as

$$
\mu_{N}=\nu_{N}+g_{\omega N} \omega_{0}+g_{\rho N} \tau_{3 N} \rho_{03}+\Sigma^{r},
$$

where $\Sigma^{r}$ is the rearrangement term that takes care of many-body effects in nuclear interaction 31,34.
We consider a second order phase transition from the nuclear to antikaon condensed phase. Nucleons in the antikaon condensed phase behave differently than nucleons in the hadronic phase 35. Kaon-nucleon interaction is considered in the same footing as the nucleon-nucleon interaction in Eq. (11). The Lagrangian density for (anti)kaons in the minimal coupling scheme is $21,35,36,37$,

$$
\mathcal{L}_{K}=D_{\mu}^{*} \bar{K} D^{\mu} K-m_{K}^{* 2} \bar{K} K
$$

where $K$ and $\bar{K}$ denote kaon and (anti)kaon doublets; the covariant derivative is $D_{\mu}=\partial_{\mu}+i g_{\omega K} \omega_{\mu}+i g_{\rho K} \mathrm{t}_{K} \cdot \boldsymbol{\rho}_{\mu}$ and the effective mass of antikaons is $m_{K}^{*}=m_{K}-g_{\sigma K} \sigma$.

The thermodynamic potential for antikaons is given by 37, 38,

$$
\frac{\Omega_{K}}{V}=T \int \frac{d^{3} p}{(2 \pi)^{3}}\left[\ln \left(1-e^{-\beta\left(\omega_{K^{-}}-\mu\right)}\right)+\ln \left(1-e^{-\beta\left(\omega_{K^{+}}+\mu\right)}\right)\right] .
$$

The in-medium energies of $K^{ \pm}$mesons are given by

$$
\omega_{K^{ \pm}}=\sqrt{\left(p^{2}+m_{K}^{* 2}\right)} \pm\left(g_{\omega K} \omega_{0}+\frac{1}{2} g_{\rho K} \rho_{03}\right)
$$

and $\mu$ is the chemical potential of $K^{-}$mesons and is given by $\mu=\mu_{n}-\mu_{p}$. The threshold condition for $K^{-}$condensation is given by $\mu=\omega_{K^{-}}=m_{K}^{*}-g_{\omega K} \omega_{0}-\frac{1}{2} g_{\rho K} \rho_{03}$.

The total (anti)kaon number density $\left(n_{K}\right)$ made of thermal kaons $\left(n_{K}^{T h}\right)$ and the $K^{-}$condensate $\left(n_{K}^{C}\right)$, is given by $n_{K}=n_{K}^{C}+n_{K}^{T}$, where

$$
\begin{aligned}
& n_{K}^{C}=2\left(\omega_{K^{-}}+g_{\omega K} \omega_{0}+\frac{1}{2} g_{\rho K} \rho_{03}\right) \bar{K} K=2 m_{K}^{*} \bar{K} K \\
& n_{K}^{T}=\int \frac{d^{3} p}{(2 \pi)^{3}}\left(\frac{1}{e^{\beta\left(\omega_{K^{-}}-\mu\right)}-1}-\frac{1}{e^{\beta\left(\omega_{K^{+}}+\mu\right)}-1}\right) \cdot(7)
\end{aligned}
$$

We calculate the pressure due to thermal kaons using $P_{K}=-\Omega_{K} / V$.

Meson field equations are solved in the mean field approximation. Meson fields are modified in the presence of $K^{-}$condensate [35, 36. Finally we obtain the pressure versus energy density known as the EoS in nuclear and antikaon condensed phases. For density dependent couplings, the pressure of nuclear matter includes the rearrangement term for thermodynamic consistency. The functional forms of nucleon-meson couplings in baryon density are given by Ref 34. The saturation density, mass of $\sigma$ meson, couplings at the saturation density and unknowns of those functions are obtained by fitting properties of finite nuclei. This parameter set is known as the DD2. Nuclear matter properties at the saturation density are in consonance with those obtained from nuclear physics experiments 14. However, kaon-meson couplings do not depend on density. In this case, kaon-vector meson coupling constants are estimated exploiting the quark model and iso-spin counting rule i.e. $g_{\omega K}=\frac{1}{3} g_{\omega N}$ and $g_{\rho K}=g_{\rho N}$ [36, 39]. The scalar coupling constant is determined from the real part of $K^{-}$optical potential at the saturation density $\left(n_{0}=0.149065 \mathrm{fm}^{-3}\right)$,

$$
U_{K^{-}}=-g_{\sigma K} \sigma_{0}-g_{\omega K} \omega_{0}+\Sigma^{r} .
$$




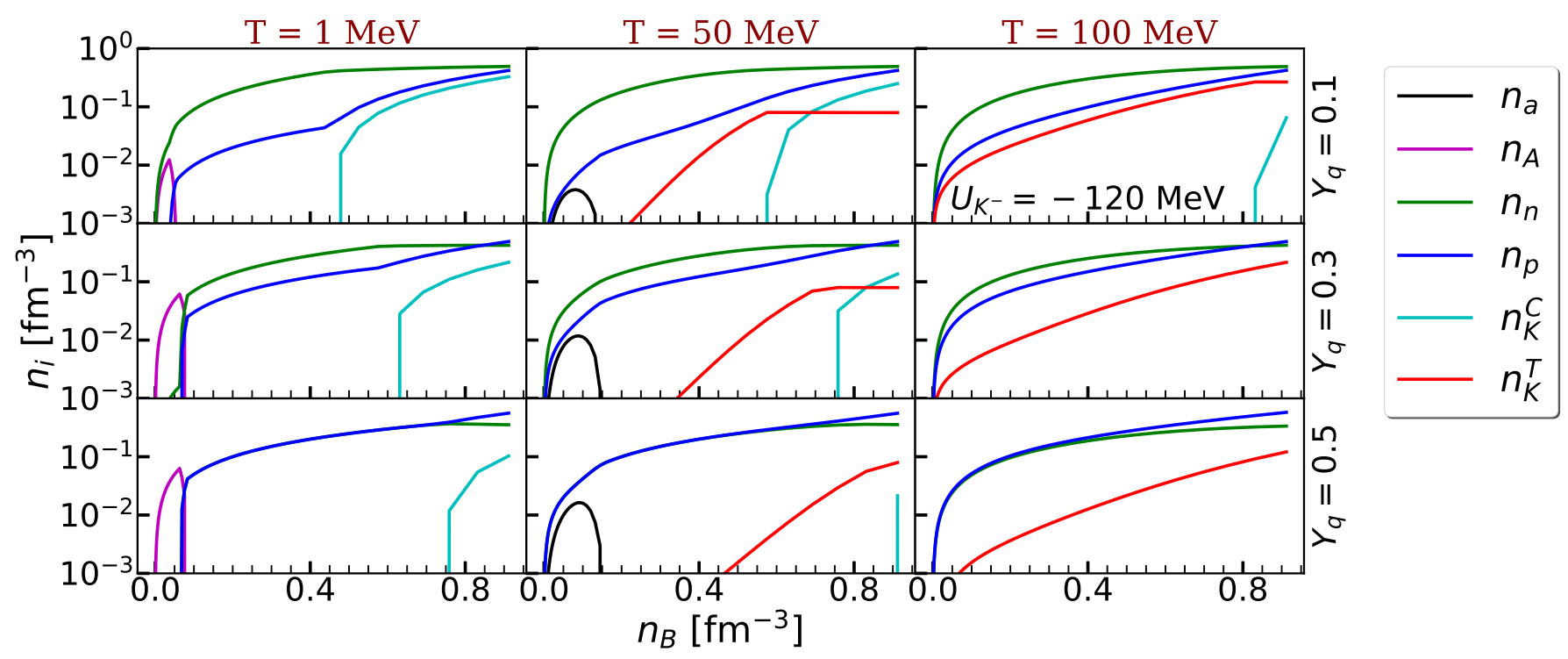

Fig. 1. Number densities of different particle species, such as light \& heavy nuclei, neutrons, protons and antikaons, both thermal and condensate, as a function of baryon number density for $\mathrm{T}=1,50,100 \mathrm{MeV}$ and $Y_{q}=0.1,0.3,0.5$.

Although the study of kaonic atoms indicates the an attractive $K^{-}$-nucleus potential, there is no consensus how deep it is. The $K^{-}$optical potential could range from -60 $\mathrm{MeV}$ to $-200 \mathrm{MeV}$ as follows from unitary chiral model calculations and phenomenological fit to kaonic atom data 40, 41,42, 43. For this calculation, we take an average antikaon optical potential depth $\mathrm{U}_{K^{-}}=-120 \mathrm{MeV}$.

\section{Results and Discussion}

We generate the EoS table with $K^{-}$condensate using the DD2 parameter set and $\mathrm{U}_{K^{-}}=-120 \mathrm{MeV}$ for a wide range of values of baryon density $\left(n_{B}\right)\left(10^{-12}\right.$ to $\left.\sim 1 \mathrm{fm}^{-3}\right)$, temperature (T) (0.1 to $158.48 \mathrm{MeV}$ ) and positive charge fraction $\left(Y_{q}\right)$ defined as $Y_{q} n_{B}=n_{p}-n_{K},(0.01$ to 0.60$)$. Grid spacings for baryon density is $\Delta \log _{10}\left(n_{B}\right)=0.04$; for temperature $\Delta \log _{10}(T)=0.04$ and for positive charge fraction $\Delta Y_{q}=0.01$. There are 301 baryon density points, 81 temperatures and 60 positive charge fractions in the EoS table. The matching of the non-uniform matter part of nucleonic HS(DD2) $\operatorname{EoS} 144$ with the kaon EoS is done in the following way. We merge two EoS tables and form a kaon EoS table including the non-uniform matter described by the extended NSE model. This merger is guided by the minimization of free energy per baryon at fixed $n_{B}, \mathrm{~T}$ and $Y_{q}$. We impose the condition that $n_{K}^{T}$ is $>10^{-4} \mathrm{fm}^{-3}$ in the non-uniform matter in addition to the above physical criterion as the contribution of antikaons to thermodynamic observables is negligible for $n_{K}^{T}<10^{-4}$ $\mathrm{fm}^{-3}$. Henceforth we call this merged EoS table 2 with $K^{-}$ as $\mathrm{HS}(\mathrm{DD} 2) \mathrm{K}^{-}$.

Populations of particles are shown for $\mathrm{T}=1,50,100$ $\mathrm{MeV}$ and $Y_{q}=0.1,0.3,0.5$ in Fig. 1. Light $(Z \leq 5)$

1 publicly available in https://compose.obspm.fr/

2 https://universe.bits-pilani.ac.in/Hyderabad/sbanik/EoS and heavy $(Z \geq 6)$ nuclei appear in non-uniform matter at temperatures $\mathrm{T}=1,50 \mathrm{MeV}$. Number densities of light and heavy nuclei are denoted by $n_{a}$ and $n_{A}$, respectively. Nuclei dissolve into uniform matter of neutrons and protons around the saturation density. It is evident from the left panel of $\mathrm{T}=1 \mathrm{MeV}$, no thermal (anti)kaons are populated in the system. The Bose-Einstein condensate of $K^{-}$mesons sets in at higher baryon densities. It is noted that the density of $K^{-}$mesons $\left(n_{K^{-}}^{C}\right)$ in the condensate is much higher for lower values of positive charge fractions for example at $Y_{q}=0.1$. Furthermore, the proton number density $\left(n_{p}\right)$ increases with the onset of the antikaon condensate and even exceeds the neutron number density $\left(n_{n}\right)$ for higher values of $Y_{q}$. The middle panel of $\mathrm{T}=50 \mathrm{MeV}$ exhibits similar features of $\mathrm{T}=1 \mathrm{MeV}$ panel with the population of thermal $\mathrm{K}^{-}$mesons. However, the density of $K^{-}$mesons in the condensate dominates over that of thermal $K^{-}$mesons $\left(n_{K^{-}}^{T h}\right)$ except for $Y_{q}=0.5$. The right panel shows the significant populations of thermal $K^{-}$mesons at $\mathrm{T}=100 \mathrm{MeV}$ for all positive charge fractions. However, in this case, the antikaon condensate disappears except for $Y_{q}=0.1$.

We have studied various thermodynamic observables such as free energy per baryon, entropy per baryon and pressure. The pressure is plotted in Fig. 2, as a function of baryon mass density. Results are shown for $\mathrm{T}=1,50,100$ $\mathrm{MeV}$ and $Y_{q}=0.1,0.3,0.5$. In all these cases, hadronic contributions are considered. The pressure does not include the contribution of leptons. It is noted that results for HS(DD2) and HS(DD2) $K^{-}$EoSs do not show any difference for $\mathrm{T}=1,50 \mathrm{MeV}$ and three positive charge fractions, at the lower density regions. However, at the higher density the antikaon condensates appear, which clearly makes the HS(DD2)K ${ }^{-}$EoS softer compared to HS(DD2) EoS. The high density portion is zoomed in the inset box for top-left panel. However we find that there are jumps in 


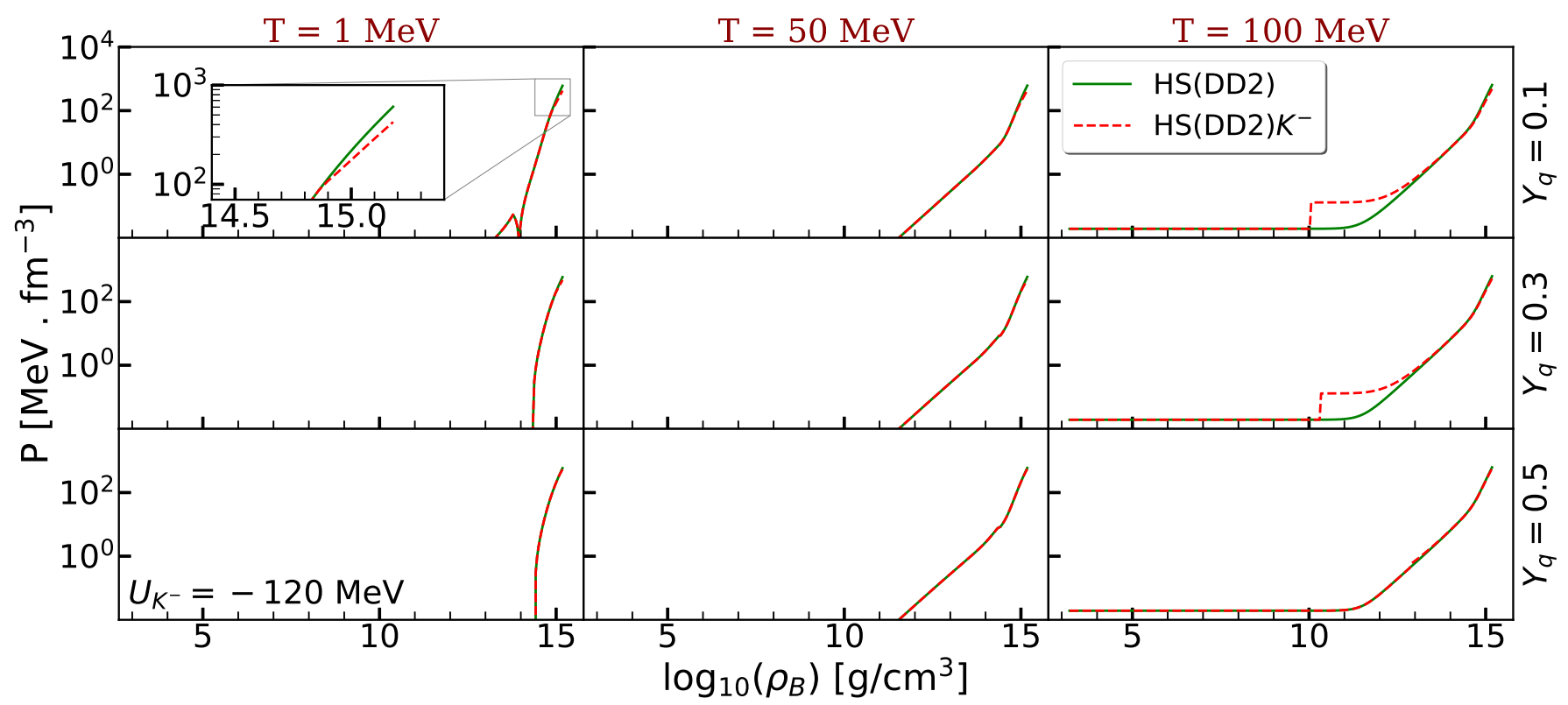

Fig. 2. Pressure is shown as a function of baryon mass density for $\mathrm{T}=1,50,100 \mathrm{MeV}$ and $Y_{q}=0.1,0.3,0.5$. The difference in the EoS at higher density is exhibited in the inset for $Y_{q}=0.1$ and $\mathrm{T}=1 \mathrm{MeV}$.

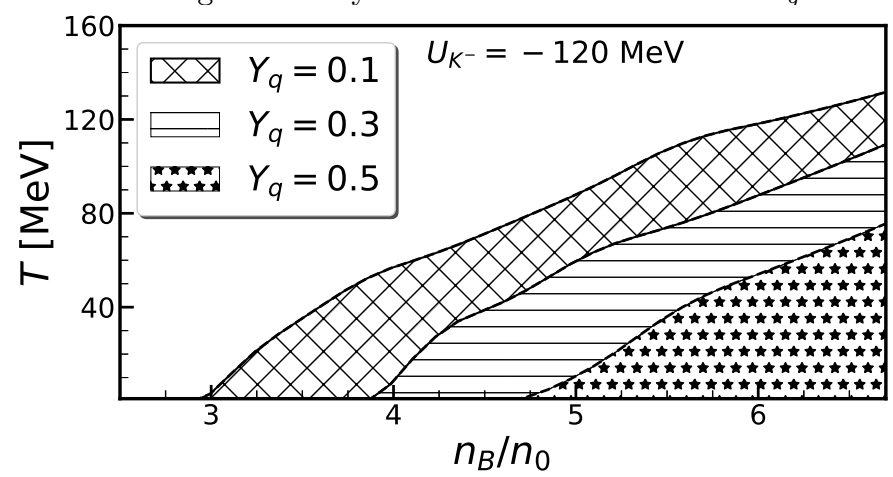

Fig. 3. The phase diagram of nuclear matter with antikaon condensate is displayed for $Y_{q}=0.1,0.3,0.5$ and $\mathrm{U}_{K^{-}}=-120$ $\mathrm{MeV}$. The $K^{-}$condensed phases, represented by the shaded regions are demarcated from the nuclear phases by the solid lines for the three $Y_{q}$ values.

pressure for $\mathrm{T}=100 \mathrm{MeV}$ and $Y_{q}=0.1$ and 0.3 due to significant contribution of thermal $K^{-}$mesons to the pressure at $\sim 10^{10} \mathrm{~g} / \mathrm{cm}^{3}$. A smooth transition from nuclear matter to $\mathrm{npK}^{-}$matter is observed for temperatures below $80 \mathrm{MeV}$.

It is expected that the antikaon condensate would cease to exist above a critical temperature $\left(T_{c}\right)$ 37. This critical temperature above which the $K^{-}$condensate dissolves, is a function of baryon density and positive charge fraction. We extract critical temperatures at different values of $n_{B}$ and $Y_{q}$ and plot the phase diagram, $T$ versus $n_{B}$, in Fig. 3. Each line in the plot denotes a particular value of $Y_{q}$. Furthermore, the region below each line represents the $K^{-}$condensate phase whereas the region above it is the nuclear phase. It is observed that the antikaon condensate region shrinks as the value of $Y_{q}$ increases.

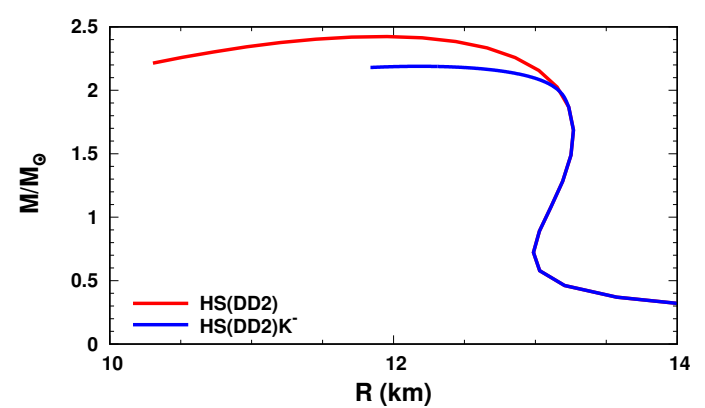

Fig. 4. Mass versus radius of a charge neutral, $\beta$-equilibrated neutron star is displayed for HS(DD2) and HS(DD2) $\mathrm{K}^{-}$with $\mathrm{U}_{K^{-}}=-120 \mathrm{MeV}$, at $\mathrm{T}=0.1 \mathrm{MeV}$.

Finally in Fig. 4. mass of neutron star is plotted with radius for charge neutral and $\beta$-equilibrated HS(DD2) and $\mathrm{HS}(\mathrm{DD} 2) \mathrm{K}^{-}$EoSs at $\mathrm{T}=0.1 \mathrm{MeV}$. It is observed that the appearance of kaon condensate make the EoS softer resulting in lower maximum mass neutron star. Maximum mass and the corresponding radii in two cases are 2.42 $\mathrm{M}_{\odot}, 11.95 \mathrm{~km}$ and $2.19 \mathrm{M}_{\odot}, 12.14 \mathrm{~km}$, respectively. The tidal deformability for $1.4 M_{\odot}$ neutron star $\left(70 \leq \Lambda_{1.4} \leq\right.$ $580)$ extracted from the BNS merger GW170817 provides radius of $1.4 M_{\odot}$ neutron star in the range $11.9_{-1.4}^{+1.4} \mathrm{~km}$ 46]. Similarly the NICER observation of PSRJ0030+0451 gives a radius of $13.02_{-1.06}^{+1.24} \mathrm{~km}$ for the $1.44 M_{\odot}$ pulsar [45]. Our results for radius corresponding to $1.4 \mathrm{M}_{\odot}$ neutron star is slightly higher than that of GW170817 [47] whereas it is consistent with the NICER observation. 


\section{Conclusions}

We have constructed a new EoS table including thermal $K^{-}$mesons and the Bose-Einstein condensate of $K^{-}$mesons known as HS(DD2) $K^{-}$for CCSN and BNS merger simulations. In this work, an extended version of the NSE model with excluded volumes is adopted for the non-uniform matter whereas the uniform matter at higher densities is described in the relativistic hadron field theory with density dependent couplings. The role of the antikaon condensate on various thermodynamic observables is investigated. The compositions of matter are appreciably modified due to the onset of the antikaon condensate at higher densities and thermal $K^{-}$at higher temperatures. The presence of the antikaon condensate makes the HS(DD2) $\mathrm{K}^{-}$ EoS softer than that of nucleonic HS(DD2) EoS. The phase diagram with the $K^{-}$condensate is also investigated. Furthermore, the charge neutral and $\beta$-equilibrated HS(DD2)K EoS results in a lower maximum mass neutron star compared with that of HS(DD2) EoS. We plan to include hyperons along with the antikaon condensate in a future publication.

\section{Acknowledgements}

Authors acknowledge the DAE-BRNS grant received under the BRNS project No.37(3)/14/12/2018-BRNS.

\section{Author Contribution}

All authors contribute equally to this work and agree to the published version of the manuscript.

\section{References}

1. M. Hempel and J. Schaffner-Bielich, Nucl. Phys. A 837, (2010) 210.

2. G. Shen, C. J. Horowitz and S. Teige, Phys. Rev. C 82, (2010) 045802, Phys. Rev. C 83, (2011) 035802, and Phys. Rev. C 83, (2011) 065808.

3. A. Steiner, M. Hempel and T. Fischer, Astrophys. J. 774, (2013) 17.

4. H. Togashi, H. Takano, K. Sumiyoshi and K. Nakazato, PTEP, 2, (2014) 023D05.

5. C. Constantinou, B. Muccioli, M. Prakash and J. M. Lattimer, Phys. Rev. C 89, (2014) 065802.

6. C. Drischler, S. Han, J. M. Lattimer, M. Prakash, S. Reddy, T. Zhao, arXiv:2009.06441.

7. M. Al-Mamun et al., arXiv:2008.12817

8. M. Marczenko, D. Blaschke, K. Redlich, C. Sasaki, arXiv:2004.09566.

9. S. Han, M. A. A. Mamun, S. Lalit, C. Constantinou, M. Prakash, Phys. Rev. D 100, (2019) 103022.

10. J. J. Li, A. Sedrakian, F. Weber, Phys. Lett. B 783, (2018) 234.

11. J. J. Li and A. Sedrakian, Astrophys. J. Lett. 874, (2019) L22, and Phys. Rev. C 100, (2019) 015809.

12. A. R. Raduta, J. J. Li and A. Sedrakian, MNRAS, 475, (2018) 4347.

13. D. Blaschke and N. Chamel in The Physics and Astrophysics of Neutron Stars, L. Rezzolla, P. Pizzochero, D. Jones, N. Rea, I. Vidaña, eds, Astrophysics and Space Science Library, 457 (2019) 337 (Springer).
14. M. Oertel, M. Hempel, T. Klähn and S. Typel 2017, Rev. Mod. Phys. 89, (2017) 015007.

15. J. Antoniadis et al., Science 340, (2013) 448.

16. H. T. Cromartie, E. Fonseca, S. M. Ransom, Nat. Astron., 4 (2019) 72.

17. G. Raaijmakers et al., Astrophys. J. Lett. 887, (2019) L22. 18. S. Weissenborn, D. Chatterjee and J. Schaffner-Bielich, Nucl. Phys. A 881, (2012) 62.

19. D. B. Kaplan \& A. E. Nelson, Phys. Lett. B 175, (1986) 57.

20. R. Knorren, M. Prakash and P. J. Ellis, Phys. Rev. C 52, (1995) 3470.

21. P. Char and S. Banik, Phys. Rev. C 90, (2014) 015801.

22. D. Chatterjee and I. Vidaña, Eur. Phys. J. A 52, (2016) 29 .

23. C. Ishizuka, A. Ohnishi, K. Tsubakihara, K. Sumiyoshi and S. Yamada, J. Phys. G 35, (2008) 085201.

24. K. Nakazato, K. Sumiyoshi and S. Yamada, Phys. Rev. D 77, (2008) 103006.

25. I. Sagert, T. Fischer, M. Hempel, G. Pagliara and J. Schaffner-Bielich, Phys. Rev. Lett. 102, (2009) 081101.

26. K. Sumiyoshi, C. Ishizuka, A. Ohnishi, S. Yamada and H. Suzuki, Astrophys. J. 690, (2009) L43.

27. H. Shen, H. Toki, K. Oyamatsu and K. Sumiyoshi, Astrophys. J. Suppl. Ser. 197, (2011) 20.

28. K. Nakazato, S. Furusawa, K. Sumiyoshi, A. Ohnishi, S. Yamada and H. Suzuki, Astrophys. J. 745, (2012) 197.

29. M. Oertel, A. F. Fantina and J. Novak, Phys. Rev. C, 85, (2012) 055806.

30. B. Peres, M. Oertel and J. Novak Phys. Rev. D 87, (2013) 043006.

31. S. Banik, M. Hempel and D. Bandyopadhyay, Astrophys. J. Suppl. Ser. 214, (2014) 22.

32. P. Char, S. Banik and D. Bandyopadhyay, Astrophys. J. 809, (2015) 116.

33. D. Radice, S. Bernuzzi, W. D. Pozzo, L. F. Roberts and C. D. Ott, Astrophys. J. Lett. 842 (2017) L10.

34. S. Typel et al., Phys. Rev. C 81, (2010) 015803.

35. N. K. Glendenning and J. Schaffner-Bielich, Phys. Rev. D 60, (1999) 025803.

36. S. Banik and D. Bandyopadhyay, Phys. Rev. C 64, (2001) 055805 .

37. S. Banik, W. Greiner and D. Bandyopadhyay, Phys. Rev. C 78, (2008) 065804.

38. J.A. Pons, S. Reddy, P.J. Ellis, M.Prakash, and J.M. Lattimer, Phys. Rev. C 62, (2020) 035803.

39. J. Schaffner and I. N. Mishustin, Phys. Rev. C 53, (1996) 1416.

40. E. Friedman, A. Gal \& C.J. Batty, Nucl. Phys. A 579, (1994) 518;

C.J. Batty, E. Friedman \& A. Gal, Phys. Rep. 287, (1997) 385.

41. E. Friedman, A. Gal, J. Mareš and A. Cieplý, Phys. Rev. C 60, (1999) 024314.

42. L. Tólos, A. Ramos and E. Oset, Phys. Rev C 74, (2006) 015203.

43. L. Tolos and L. Fabbietti, Prog. Part. Nucl. Phys. 112, (2020) 130770.

44. T. Fischer, M. Hempel, I. Sagert, Y. Suwa and J. SchaffnerBielich, Eur. Phys. J. A, 50, (2014) 46.

45. M. C. Miller et al., Astrophys. J. Lett. 887, (2019) L24.

46. B. P. Abbott et al., Phys. Rev. Lett. 121 (2018) 161101.

47. S. Soma and D. Bandyopadhyay, Astrophys. J. 890 (2020) 139. 
